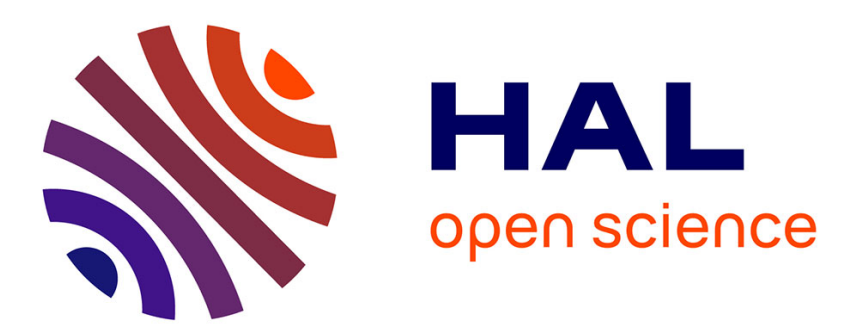

\title{
Influence des conditions initiales sur l'évolution d'une onde de choc induite par impulsion laser \\ F. Cottet, J.P. Romain
}

\section{To cite this version:}

F. Cottet, J.P. Romain. Influence des conditions initiales sur l'évolution d'une onde de choc induite par impulsion laser. Journal de Physique Lettres, 1983, 44 (7), pp.235-240. 10.1051/jphyslet:01983004407023500 . jpa-00232186

\section{HAL Id: jpa-00232186 https://hal.science/jpa-00232186}

Submitted on 1 Jan 1983

HAL is a multi-disciplinary open access archive for the deposit and dissemination of scientific research documents, whether they are published or not. The documents may come from teaching and research institutions in France or abroad, or from public or private research centers.
L'archive ouverte pluridisciplinaire HAL, est destinée au dépôt et à la diffusion de documents scientifiques de niveau recherche, publiés ou non, émanant des établissements d'enseignement et de recherche français ou étrangers, des laboratoires publics ou privés. 


\title{
LE JOURNAL DE PHYSIQUE-LETTRES
}

J. Physique - LETTRES 44 (1983) L-235 - L-240

1er AVRIL 1983, PAGE L-235

Classification

Physics Abstracts

$52.40 \mathrm{H}-62.50$

\section{Influence des conditions initiales sur l'évolution d'une onde de choc induite par impulsion laser}

\author{
F. Cottet et J. P. Romain \\ Laboratoire d'Energétique et Détonique, LA 193, E.N.S.M.A., 86034 Poitiers Cedex, France \\ et GRECO Interaction Laser Matière, Laboratoire PMI, Ecole Polytechnique, 91128 Palaiséau, \\ France
}

(Reçu le 28 octobre 1982, révisé le 26 janvier 1983, accepté le 15 février 1983)

\begin{abstract}
Résumé. - L'évolution d'un choc de forte amplitude induit par une impulsion laser dans une cible solide est décrite à l'aide d'un modèle hydrodynamique, précisant un modèle antérieur par la prise en compte du déplacement de la surface avant de la cible sous l'effet de la pression d'ablation. Ce modèle est également utilisé pour étudier l'influence du profil initial de pression sur l'amortissement de l'onde de choc au cours de sa propagation.
\end{abstract}

\begin{abstract}
The evolution of a high amplitude laser-generated shock in a solid target is described by the use of a hydrodynamic model. In comparison with a previous model, this one takes into account the front face target propagation due to the ablation pressure effect. This model is also used to study the initial pressure profile effect on the shock wave decay.
\end{abstract}

L'interaction d'une impulsion laser de forte puissance avec une cible solide produit un plasma dont la détente exerce, par réaction, une compression du solide sous la forme d'une onde de choc de très forte amplitude [1]. La durée des impulsions laser utilisées étant très faible, les ondes de choc induites s'amortissent très rapidement au cours de leur propagation dans la cible [2-4]. Il est donc nécessaire de prendre en compte cet effet pour évaluer la pression maximale induite, correspondant à la pression d'ablation, à partir des mesures de l'amplitude du choc à différentes profondeurs dans la cible.

L'évolution de la pression à la surface d'ablation peut être modélisée par un profil trapézoïdal (Fig. 1) : montée en pression linéaire d'amplitude maximale $P_{\mathrm{M}}$ pendant un temps $T_{1}$, maintien en pression pendant un temps $T_{2}$ et décroissance linéaire de durée $T_{3}$ ramenant le milieu à une pression nulle. On adopte une largeur à mi-hauteur $\tau$ égale à celle de l'impulsion laser :

$$
\tau=T_{2}+\frac{T_{1}+T_{3}}{2}
$$




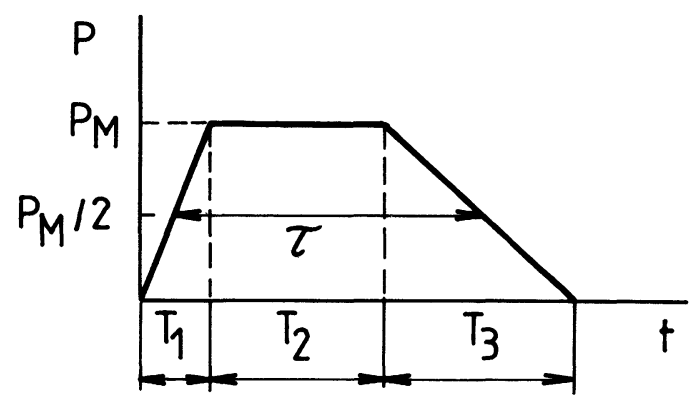

Fig. 1. - Modélisation de la variation de pression appliquée sur la cible en fonction du temps : $\boldsymbol{P}_{\mathrm{M}}:$ pression maximale, $T_{1}$ : temps de montée, $T_{2}$ : temps de maintien, $T_{3}$ : temps de décroissance de la pression, $\tau$ : largeur à mi-hauteur.

[Pressure profile applied on the surface target as a function of time. $P_{\mathrm{M}}:$ maximum pressure, $T_{1}:$ rise time, $T_{2}$ : duration of steady pressure, $T_{3}:$ decay time, $\tau$ : width at half maximum.]

Les différentes phases de l'évolution de la pression dans la cible, résultant d'effets hydrodynamiques, sont : formation d'une onde de choc, croissance de l'amplitude du choc, maintien puis amortissement de l'onde de choc. Dans le repère du laboratoire, la vitesse des ondes de compression ou de détente derrière le choc est la somme de la vitesse du son locale $C$ et de la vitesse matérielle $u$. La célérité du choc $D$ étant inférieure à la somme « $C+u$ », les ondes de compression ou de détente rattrapent l'onde de choc dont l'amplitude varie en conséquence.

1. Equation générale de l'évolution d'une onde de choc résultant d'effets hydrodynamiques. La dérivée d'une grandeur $f(X, t)$ liée au front de choc s'exprime par :

$$
\frac{\mathrm{D} f}{\mathrm{D} t}=\frac{\partial f}{\partial t}+D \frac{\partial f}{\partial X}
$$

où $D=\mathrm{D} X / \mathrm{D} t$ représente la célérité du front de choc, et la dérivée d'une grandeur $f(X, t)$ liée à un point matériel du plan par :

$$
\frac{\mathrm{d} f}{\mathrm{~d} t}=\frac{\partial f}{\partial t}+u \frac{\partial f}{\partial X}
$$

où $u=\mathrm{d} X / \mathrm{d} t$ représente la vitesse d'un point matériel.

La mise en équation se fait en appliquant les lois de conservation de la quantité de mouvement :

$$
\rho \frac{\mathrm{d} u}{\mathrm{~d} t}+\frac{\partial P}{\partial X}=0
$$

et de la conservation de la masse :

$$
\frac{\mathrm{d} P}{\mathrm{~d} t}+\rho \frac{\mathrm{d} u}{\mathrm{~d} t}+\frac{(n-1) \rho u}{X}=0
$$

où $\rho$ représente la masse volumique du matériau de valeur initiale $\rho_{0}$ et $n$ caractérise la géométrie : $n=1$ en géométrie plane et $n=3$ en géométrie sphérique.

En supposant le front d'onde de choc discontinu et l'écoulement isentropique derrière le choc, 
les équations (2) et (3) permettent d'obtenir l'expression de la variation d'amplitude de l'onde de choc $P$ en fonction de la distance $X[5]$ :

$$
D\left((D-u)+\rho C^{2} \frac{\mathrm{d} u}{\mathrm{~d} P}\right) \frac{\mathrm{D} P}{\mathrm{D} X}=\left((D-u)^{2}-C^{2}\right) \frac{\partial P}{\partial X}-\frac{(n-1) C^{2} u \rho(D-u)}{X}
$$

où $\partial P / \partial X$ représente le gradient de pression derrière le front de choc.

Les conditions initiales, définies par le profil de pression appliquée (Fig. 1), étant données en coordonnées pression-temps, on transforme l'équation précédente (4) dans le même système de coordonnées par la relation :

$$
\frac{\partial P}{\partial X}=\frac{1}{D}\left(\frac{\mathrm{D} P}{\mathrm{D} t}-\frac{\partial P}{\partial t}\right)
$$

On obtient ainsi le système d'équations permettant de calculer l'évolution de la pression de choc $P$ en fonction du temps $t$ et de la distance $X[6-7]$ :

$$
\left\{\begin{array}{l}
\frac{\mathrm{D} P}{\mathrm{D} t}=-A_{1} \frac{\partial P}{\partial t}-\frac{A_{2}}{X+d} \\
\text { avec }: \\
A_{1}=\frac{(D-u)^{2}-C^{2}}{(D-u) D+\rho_{0} \frac{D^{2} C^{2}}{(D-u)} \frac{\mathrm{d} u}{\mathrm{~d} P}+C^{2}-(D-u)^{2}} \\
A_{2}=\frac{(n-1) \rho_{0} D u C^{2}}{(D-u)+\rho_{0} \frac{D C^{2}}{(D-u)} \frac{\mathrm{d} u}{\mathrm{~d} P}+\frac{C^{2}-(D-u)^{2}}{D}} \\
\frac{\mathrm{D} X}{\mathrm{D} t}=D,
\end{array}\right.
$$

où $X$ représente la position du front de choc d'amplitude $P$ à l'instant $t$ par rapport à l'état initial, $\partial P / \partial t$ représente la pente derrière le front de choc et $d$ représente le diamètre de la tache focale du faisceau laser. La géométrie sphérique est prise en compte dans la phase de décroissance de l'onde de choc à partir d'une distance égale à $d$ et avec un rayon de courbure de valeur initiale $d$. Cette condition simplificatrice arbitraire est justifiée par la faible distance à laquelle le choc atteint son amplitude maximale. Elle n'intervient que pour les taches focales de faible diamètre.

Les expressions de $\dot{D}, u$ et $C$, en fonction de la pression, sont obtenues à partir de la courbe de Hugoniot et de l'équation d'état du matériau, de la forme équation d'état de Mie-Gruneisen [7]. La valeur de la vitesse du son est déduite de l'expression générale donnée par McQueen [8], en faisant l'hypothèse classique pour le coefficient de Gruneisen $\gamma$ de valeur initiale $\gamma_{0}$ :

$$
\gamma \rho=\gamma_{0} \rho_{0} .
$$

Les données relatives au matériau et introduites dans le calcul, sont la densité initiale $\rho_{0}$, le coefficient de Gruneisen $\gamma_{0}$ [8] et les coefficients $C_{0}$ et $S$ de la relation linéaire expérimentale entre $D$ et $u$, [9] :

$$
D=C_{0}+S u
$$




\section{Calcul de la pente derrière le front de choc.}

L'expression de $\partial P / \partial t$ est obtenue en considérant que les éléments d'onde de compression ou de détente successifs rattrapent le front de choc, en partant de la surface avant soumise à une évolution de pression en fonction du temps décrite par le profil de pression de la figure 1 .

Le principe de calcul de la pente derrière le front de choc $\partial P / \partial t$ en phase de croissance est décrit par la figure 2. L'onde de choc atteint la pression $P_{\mathrm{i}}$ à l'instant $T_{\mathrm{i}}$ et à la distance $X_{\mathrm{i}}$, coordonnées du point où la caractéristique $(\delta)$ de pression $P_{\mathrm{i}}$ issue de la surface avant de la cible au point de coordonnées $\left(X_{\mathrm{i} 0}, T_{\mathrm{i} 0}\right)$ rejoint la trajectoire $(\alpha)$ du front de choc. La caractéristique suivante $\left(\delta^{\prime}\right)$ à la pression $P_{\mathrm{i}}+\Delta P$, issue du point $\left(X_{\mathrm{i} 0}^{\prime}, T_{\mathrm{i} 0}^{\prime}\right)$ atteint la distance $X_{\mathrm{i}}$ à l'instant $T_{\mathrm{i}}^{\prime}$. On a alors :

où

$$
\begin{gathered}
\frac{\partial P}{\partial t}=\lim _{\Delta P \rightarrow 0} \frac{\Delta P}{T_{\mathrm{i}}^{\prime}-T_{\mathrm{i}}}, \\
T_{\mathrm{i}}=T_{\mathrm{i} 0}+\frac{X_{\mathrm{i}}-X_{\mathrm{i} 0}}{(C+u)_{P_{\mathrm{i}}}} \\
T_{\mathrm{i}}^{\prime}=T_{\mathrm{i} 0}^{\prime}+\frac{X_{\mathrm{i}}-X_{\mathrm{i} 0}^{\prime}}{(C+u)_{P_{\mathrm{i}}+\Delta P}},
\end{gathered}
$$

avec

$$
X_{\mathrm{i} 0}^{\prime}=X_{\mathrm{i} 0}+\left(T_{\mathrm{i} 0}^{\prime}-T_{\mathrm{i} 0}\right) u_{P_{\mathrm{i}}},
$$

où $u_{P_{\mathrm{i}}}$ représente la vitesse matérielle à la pression $P_{\mathrm{i}}$, pente de la trajectoire $(\gamma)$ de la surface avant de la cible.

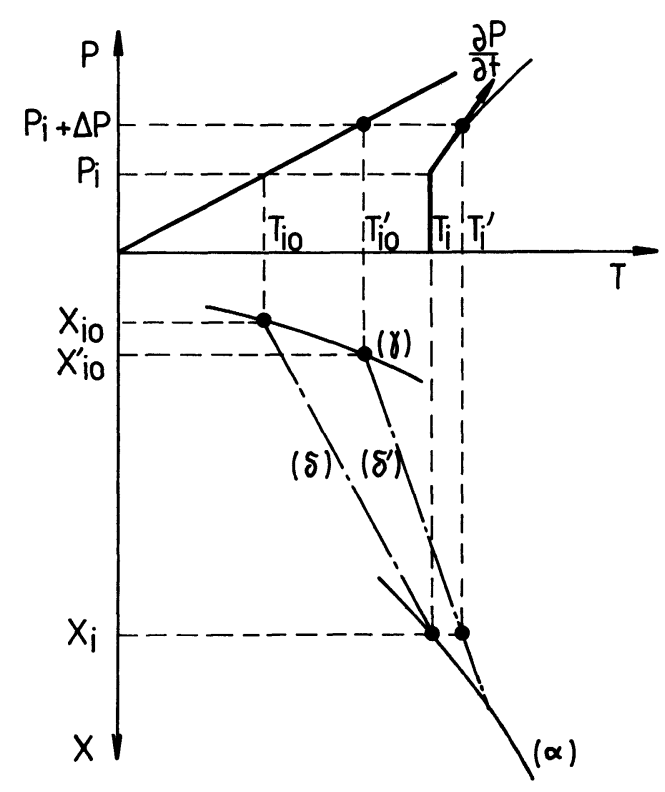

Fig. 2. - Evolution de la pente derrière le front de choc dans la phase de croissance de l'onde de choc; $(\alpha)$ trajectoire du front de choc; $(\gamma)$ trajectoire de la face avant de la cible; $(\delta)$ et $\left(\delta^{\prime}\right)$ caractéristiques de pression $\boldsymbol{P}_{\mathrm{i}}$ et $\boldsymbol{P}_{\mathrm{i}}+\Delta \boldsymbol{P}$ respectivement.

[Evolution of the pressure gradient behind the shock front in the region of increasing shock pressure ; $(\alpha)$ shock front trajectory; $(\gamma)$ front face target trajectory; $(\delta)$ and $\left(\delta^{\prime}\right)$ compressional waves taking the solid respectively to the pressure $P_{\mathrm{i}}$ and $P_{\mathrm{i}}+\Delta P$.] 
Compte tenu de la variation initiale linéaire de la pression, adoptée dans le modèle utilisé, on a :

$$
T_{\mathrm{i} 0}^{\prime}-T_{\mathrm{i} 0}=\frac{T_{1}}{P_{\mathrm{M}}} \Delta P .
$$

En reportant les relations (8) à (11) dans la relation (7), on obtient ainsi :

$$
\begin{aligned}
\frac{\partial P}{\partial t}=\lim _{\Delta P \rightarrow 0}\left[\frac{T_{1}}{P_{M}}(1\right. & \left.-\frac{u_{P_{1}}}{(C+u)_{P_{1}+\Delta P}}\right)- \\
& \left.-\left(X_{\mathrm{i}}-X_{\mathrm{i} 0}\right) \frac{(C+u)_{P_{\mathrm{i}}+\Delta P}-(C+u)_{P_{\mathrm{i}}}}{\Delta P} \frac{1}{(C+u)_{P_{\mathrm{i}}}(C+u)_{P_{\mathrm{i}}+\Delta P}}\right]^{-1},
\end{aligned}
$$

soit :

$$
\frac{\partial P}{\partial t}=\left[\frac{T_{1}}{P_{\mathrm{M}}} \frac{C}{C+u}-\frac{X_{\mathrm{i}}-X_{\mathrm{i} 0}}{(C+u)^{2}}\left(\frac{\mathrm{d} C}{\mathrm{~d} P}+\frac{\mathrm{d} u}{\mathrm{~d} P}\right)\right]^{-1} .
$$

Cette expression est valable en phase de croissance de l'onde de choc. Un calcul identique, effectué en phase de décroissance, conduit à l'expression suivante :

$$
\frac{\partial P}{\partial t}=-\left[\frac{T_{3}}{P_{\mathrm{M}}} \frac{C}{C+u}+\frac{X_{\mathrm{i}}-X_{\mathrm{i} 0}}{(C+u)^{2}}\left(\frac{\mathrm{d} C}{\mathrm{~d} P}+\frac{\mathrm{d} u}{\mathrm{~d} P}\right)\right]^{-1} .
$$

$\partial P / \partial t$ peut être ainsi calculé en fonction de la pression, à partir des valeurs connues de $C$, de $u$ et de leurs dérivées sur la courbe de Hugoniot du milieu étudié. Tous les éléments du système d'équations (5) et (6) sont alors déterminés et la résolution numérique de ce système est effectuée par la méthode d'Euler. Les conditions initiales du calcul sont définies, pour un matériau donné, par le diamètre de la tache focale, la pression maximale induite et la valeur des paramètres $T_{1}$, $T_{2}, T_{3}$ liés à l'impulsion laser.

Dans un calcul antérieur [7] relatif à l'évaluation de $\partial P / \partial t$, le déplacement de la surface avant de la cible avait été négligé. Cette hypothèse simplificatrice introduit une erreur que nous avons pu estimer en effectuant des simulations numériques pour des conditions initiales identiques, mais en tenant compte du déplacement de la surface. L'écart est d'environ $8 \%$ pour une pression maximale $P_{\mathrm{M}}$ de $500 \mathrm{kbar}$, et environ $30 \%$ à $5 \mathrm{Mbar}$, en impulsion laser de durée $\tau=3,5 \mathrm{~ns}$ et en géométrie plané.

\section{Influence du profil de pression appliquée sur l'évolution de la pression de choc dans la cible.}

Les conditions initiales introduites dans le calcul sont déterminées par le profil trapézoïdal de pression appliquée modélisant le profil réel. Les paramètres liés à l'impulsion laser (pression maximale induite $\boldsymbol{P}_{\mathrm{M}}$ et largeur à mi-hauteur $\tau$ de l'impulsion) étant fixés, on peut cependant faire varier le temps de montée $T_{1}$, le temps de maintien $T_{2}$ et le temps de chute $T_{3}$ de la pression appliquée.

La figure 3 montre les effets d'une variation de ces paramètres sur l'évolution calculée de l'amplitude du choc dans l'interprétation des résultats expérimentaux obtenus précédemment pour des impulsions laser de durée à mi-hauteur $3,5 \mathrm{~ns}$ et un flux incident de $3 \times 10^{12} \mathrm{~W} / \mathrm{cm}^{2}[7$, 10]. Compte tenu de l'incertitude sur les mesures, on constate que les courbes calculées, entre le profil triangulaire d'une part et un profil à temps de montée et temps de chute très faibles d'autre part, restent compatibles avec les résultats expérimentaux. La pression maximale de 1,2 Mbar, obtenue par extrapolation des mesures à l'aide des courbes calculées, ne dépend donc pratiquement pas de la forme du profil initial utilisé pour le calcul. 


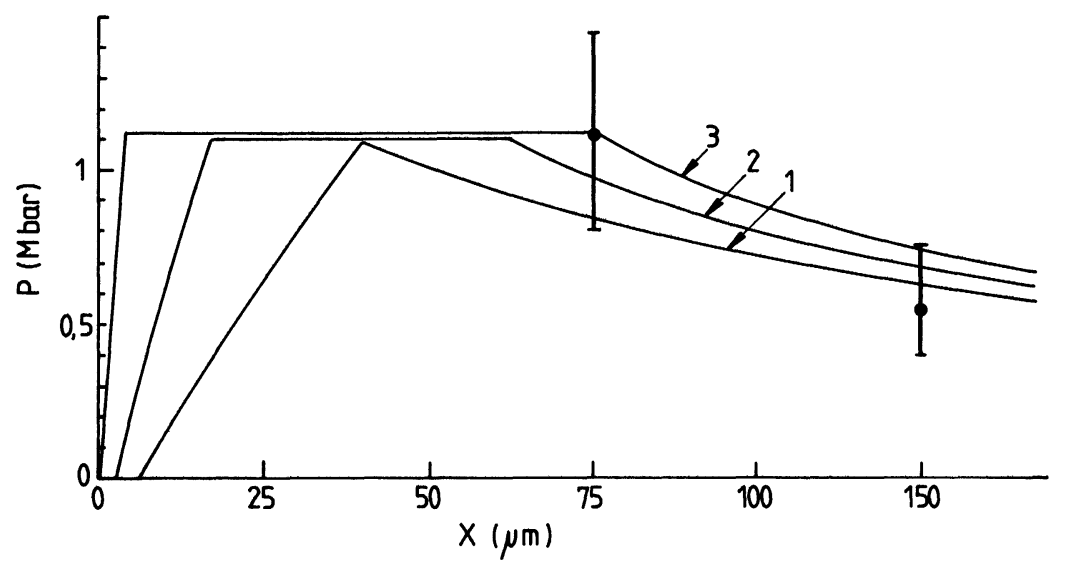

Fig. 3. - Evolution calculée de la pression de choc dans une cible de cuivre pour différents profils symétriques de pression appliquée. La pression maximale, $P_{\mathrm{M}}=1,2 \mathrm{Mbar}$, et la largeur à mi-hauteur, $\tau=3,5 \mathrm{ns,}$ sont fixées. Courbe 1: $T_{1}=T_{3}=3,5 \mathrm{~ns} ; T_{2}=0$. Courbe 2: $T_{1}=T_{3}=1,5 \mathrm{~ns} ; T_{2}=2 \mathrm{~ns}$. Courbe 3: $T_{1}=T_{3}=0,5 \mathrm{~ns} ; T_{2}=3 \mathrm{~ns}$. Les points expérimentaux ont été obtenus avec des impulsions laser de durée à mi-hauteur $3,5 \mathrm{~ns}$ et un flux incident de $3 \times 10^{12} \mathrm{~W} / \mathrm{cm}^{2}[7-10]$.

[Calculated shock pressure evolution in a copper target for various applied pressure profiles. The maximum pressure, $P_{\mathrm{M}}=1.2 \mathrm{Mbar}$, and the width at half maximum, $\tau=3.5 \mathrm{~ns}$, are fixed. Curve $1: T_{1}=T_{3}=3.5 \mathrm{~ns}$; $T_{2}=0$. Curve 2: $T_{1}=T_{3}=1.5 \mathrm{~ns} ; T_{2}=2 \mathrm{~ns}$. Curve 3: $T_{1}=T_{3}=0.5 \mathrm{~ns} ; T_{2}=3 \mathrm{~ns}$. Experimental results were obtained with a laser pulse of $3.5 \mathrm{~ns}$ FWHM and an incident intensity of $3 \times 10^{12} \mathrm{~W} / \mathrm{cm}^{2}$ [7-10].]

En résumé, ce résultat montre que l'incertitude sur la détermination de la pression induite, due à l'approximation sur le profil de pression appliquée, peut être négligée par rapport aux incertitudes expérimentales sur les mesures de pression. Par contre, l'erreur commise, en négligeant le déplacement de la surface initiale dans le calcul de la pente derrière le front de choc, est importante aux fortes pressions.

\section{Bibliographie}

[1] Trainor, R. J., Shaner, J. W., Auerbach, J. M., Holmes, N. C., Phys. Rev. Lett. 42 (1979) 1154.

[2] Trainor, R. J., Graboske, H. C., Long, K. S. and Shaner, J. W., Report of Lawrence Livermore Laboratory UCRL 52562 (1978).

[3] Veeser, L. M., Solem, J. C., Lieber, A. J., Appl. Phys. Lett. 35(1979) 761.

[4] Romain, J. P. and Cotret, F., High Pressure Science \& Technology, ed. by B. Vodar \& Ph. Marteau (Pergamon Press) 1980, p. 968.

[5] Duvall, G. E., Iran. J. Sci. Technol. 7 (1978) 57.

[6] Cotret, F., Thèse de spécialité $\mathbf{n}^{0} 794$, Poitiers (1981).

[7] Cottet, F. and Romain, J. P., Phys. Rev. A 25 (1982) 576.

[8] MCQueen, R. G., Marsh, S. P., TAYlor, J. W., Fritz, J. N., and CARTer, W. J., High Velocity Impact Phenomena, ed. by R. Kinslow (Academic Press, New York) 1970.

[9] Mitchell, A. C. and Nellis, W. J., J. Appl. Phys. 52 (1981).

[10] Cottet, F. and Romain, J. P., High Pressure in Research and Industry, ed. by C. M. Bachman, T. Johannison and L. Tegner (Uppsala, Sweden) 1982, p. 168. 